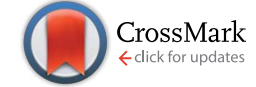

Cite this: RSC Adv., 2017, 7, 9472

Received 12th December 2016 Accepted 25th January 2017

DOI: $10.1039 / c 6 r a 28079 j$

rsc.li/rsc-advances

\section{Dioxetane formation and chemiluminescent emission upon the combination of a vinylphenol derivative with naphthalene endoperoxide $\uparrow$}

\author{
Yui Umehara, ${ }^{a}$ Aoi Son, ${ }^{a}$ Teruyuki Kondo*a and Kazuhito Tanabe ${ }^{* b}$
}

\begin{abstract}
We designed a chemiluminescent system using nanoparticles. We prepared mesoporous nanoparticles bearing naphthalene endoperoxide as a singlet oxygen generator in the pore and vinyl phenol derivative as an emission unit. The combination of these functional molecules gave highly efficient and bright chemiluminescence through the formation of a 1,2-dioxetane intermediate.
\end{abstract}

Chemiluminescent technology offers considerable potential for biological imaging, and various systems have been developed to characterize biological functions and properties. ${ }^{1,2}$ In particular, the combination of luciferase and luciferin, which produces red emission through the consumption of ATP, allows biological processes to be visualized in living cells and animals. ${ }^{3-12}$ However, the practical use of conventional chemiluminescent techniques has been limited because they have several drawbacks, such as requirements for complex gene technology and cell manipulation to generate specific enzymes in the subjects, resulting in relatively low versatility. On the other hand, small-molecule-based chemiluminescent systems that are based on distorted dioxetane derivatives have been developed. ${ }^{13-15}$ However, their suitability for biological imaging is also limited because of the instability of the molecules and the difficulties involved in further modification of the core structure. Therefore, much effort has recently been made to identify simpler chemiluminescent systems.

Herein, we report a novel chemiluminescent system in which dioxetane derivatives are formed in situ. We developed this system using a combination of naphthalene endoperoxide (NEP), which generates singlet oxygen $\left({ }^{1} \mathrm{O}_{2}\right)$ through thermolysis, and vinylphenols bearing an adamantyl group (VPA) as an emission unit. The latter is converted into dioxetane derivatives through the $[2+2]$ cycloaddition of ${ }^{1} \mathrm{O}_{2}$ (Fig. 1). Mixing of VPA with NEP leads to the spontaneous formation of dioxetane and

\footnotetext{
${ }^{a}$ Department of Energy and Hydrocarbon Chemistry, Graduate School of Engineering, Kyoto University, Katsura, Nishikyo-ku, Kyoto 615-8510, Japan. E-mail: teruyuki@scl. kyoto-u.ac.jp; Fax: +81-75-383-2504; Tel: +81-75-383-7055

${ }^{b}$ Department of Chemistry and Biological Science, College of Science and Engineering, Aoyama Gakuin University, 5-10-1 Fuchinobe, Chuo-ku, Sagamihara, 252-5258, Japan. E-mail: tanabe.kazuhito@chem.aoyama.ac.jp; Fax: +81-42-759-6493; Tel: +81-42759-6229

$\dagger$ Electronic supplementary information (ESI) available: Detail of the experimental procedure and MS spectra of VPA, absorption spectra of MSN-NEP and HPLC profile of VPA before and after the reaction. See DOI: $10.1039 / \mathrm{c} 6 \mathrm{ra} 28079 \mathrm{j}$
}

the emission of chemiluminescence without any additives or other triggers. Furthermore, we prepared mesoporous nanoparticles bearing NEP within their pores (MSN-NEP) and confirmed that these particles induced the oxidation of VPA and led to chemiluminescence. The present system is a promising approach to chemiluminescence-based bio-imaging without the need for either gene or cellular manipulation.

Initially, we prepared VPA and characterized its properties as an emission unit, because it is known to be a representative precursor of a chemiluminescent emission unit. ${ }^{13}$ On the other hand, NEP, as a donor of ${ }^{1} \mathrm{O}_{2}$, was prepared from 1,4-dimethylnaphthalene by the photosensitization of methylene blue. To evaluate the chemiluminescence of VPA, we combined VPA and NEP and monitored the emission. As shown in Fig. 2A, we observed chemiluminescent emission at around $450 \mathrm{~nm}$ that was typical of oxidized VPA, indicating that VPA was spontaneously converted into the corresponding dioxetane derivative in the presence of ${ }^{1} \mathrm{O}_{2}$ generated from the thermal degradation of NEP. The luminescence of VPA increased as the $\mathrm{pH}$ was increased, suggesting that deprotonation of the hydroxyl group on the aromatic unit is a key for the emission of VPA, which is consistent with previous reports. ${ }^{13}$ We also confirmed that the emission of VPA in the presence of NEP could be seen with the naked eye, as shown in Fig. 2B.

To evaluate this reaction in detail, we next analyzed the products obtained from $[2+2]$ cycloaddition of VPA and ${ }^{1} \mathrm{O}_{2}$ generated from NEP by means of GC-MS. Although the formation of dioxetane derivative of VPA was not detected, the measurement of MS spectra indicated the formation of methyl 3-hydroxybenzoate (molecular weight: 152), which was formed by the degradation of dioxetane derivative (Fig. S1 $\dagger$ ). This result strongly supports the occurrence of cycloaddition to form the dioxetane derivative.

It is well known that ${ }^{1} \mathrm{O}_{2}$ is a strong oxidant that can act on a huge variety of compounds including organic molecules, polymers as well as biomolecules. Thus, control of its activity is 


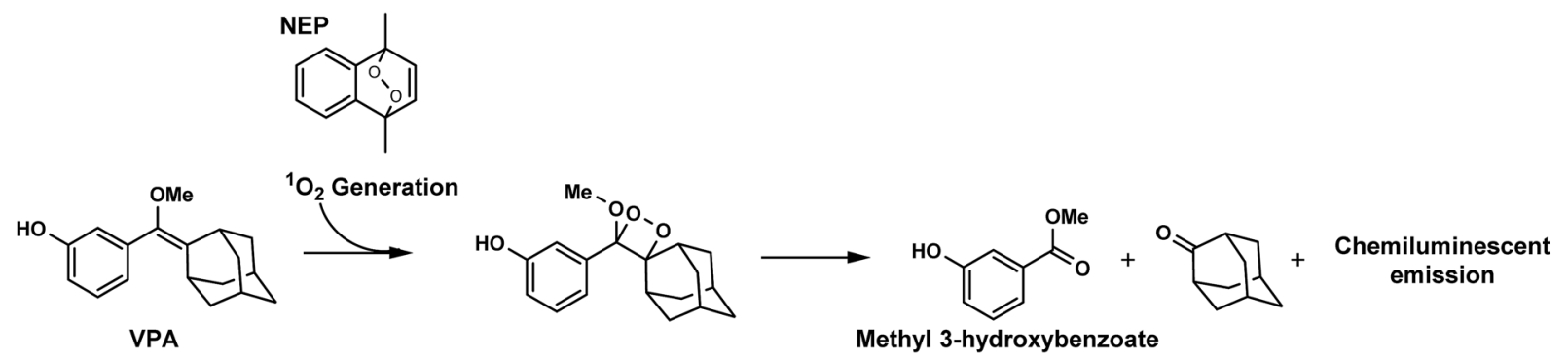

Fig. 1 Schematic outline of chemiluminescence system using VPA and thermal ${ }^{1} \mathrm{O}_{2}$ generation from NEP.

(A)

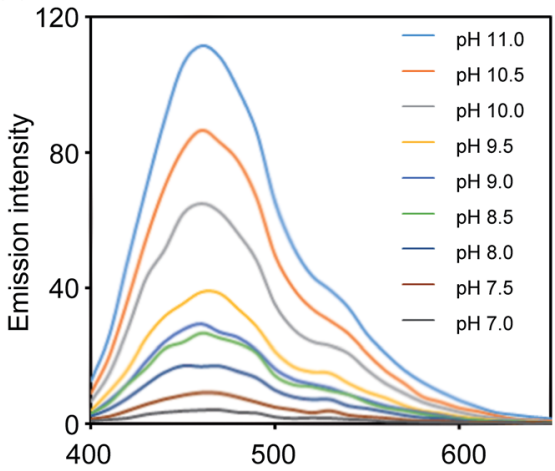

Fig. 2 (A) Chemiluminescent spectra of VPA $(1.5 \mathrm{mM})$ in the presence of NEP $(20 \mathrm{mM})$. The spectra were recorded under various $\mathrm{pH}$ conditions. (B) Visualization of chemiluminescence emission of VPA in the presence of NEP ( $\mathrm{pH}$ 11.0).

critical for the practical use of the present system in bioimaging. It has been proposed that ${ }^{1} \mathrm{O}_{2}$ species generated in the pores of nanoparticles are confined within the pores and inactivated before leaking out because of their short lifetime. ${ }^{\mathbf{1 6}}$
Therefore, we designed a system in which NEP was incorporated within the pores of mesoporous silica nanoparticles (MSNNEP). The synthesis of MSN-NEP is outlined in Scheme 1. Acid 1 was coupled with an ethylenediamine derivative to form amide 2. Removal of the Boc group in 2 under acidic conditions gave naphthalene derivative 3 possessing a primary amino group. Coupling of MSN bearing carboxylic acid groups (MSN$\mathrm{CO}_{2} \mathrm{H}$ ) with 3 formed nanoparticles possessing naphthalene units (MSN-NA). The photooxidation of MSN-NA in the presence of methylene blue as a photochemical generator of ${ }^{1} \mathrm{O}_{2}$ furnished MSN-NEP. The formation of endoperoxide at the naphthalene unit was confirmed by measurement of the absorption spectrum. As shown in Fig. 3A, the absorption at around $300 \mathrm{~nm}$ in MSN-NA, which was assigned to absorption by the naphthalene unit, decreased as the photooxidation time increased, which indicated that NEP was formed in pores. In order to identify the thermal generation of singlet oxygen $\left({ }^{1} \mathrm{O}_{2}\right)$ from MSN-NEP, we measured the phosphorescence of ${ }^{1} \mathrm{O}_{2}$ formed from MSN-NEP. We conducted photo-irradiation of MSN-NEP to generate heat for the formation of ${ }^{1} \mathrm{O}_{2}$ and measured the emission of ${ }^{1} \mathrm{O}_{2}$. We observed the emission at $1270 \mathrm{~nm}$, which was identified as the phosphorescence of ${ }^{1} \mathrm{O}_{2}$. In addition, we<smiles>Cc1ccc(CCC(=O)NCCNC(=O)NCCNC(C)(C)C)c2ccccc12</smiles>

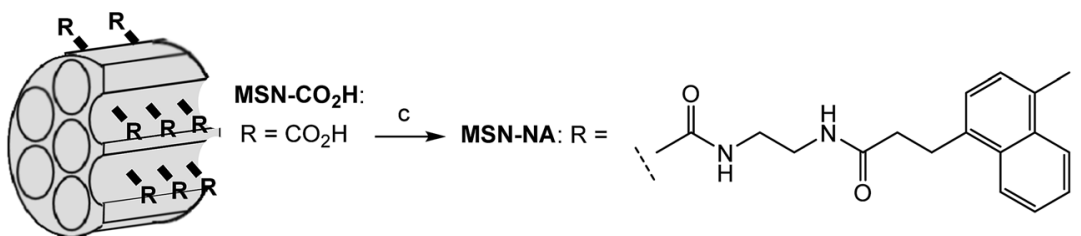<smiles>CI(C=P[As])C(=O)NCCNC(=O)CCC12C=CC(C)(O1)C1C=CC=CC12</smiles>

Scheme 1 Synthesis of MSN-NEP. Reagents and conditions. (a) EDCl, HOBt, DMF, 74\%; (b) HCl, 15\%; (c) 3, EDCl, HOBt, DMF; (d) hv (665 nm), methylene blue. 

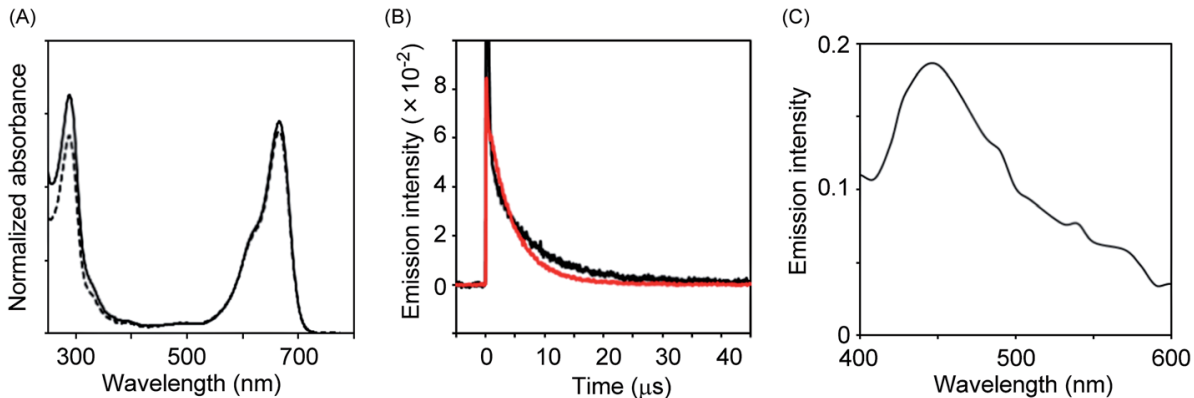

Fig. 3 (A) Absorption spectra of MSN-NA before or after oxidation by ${ }^{1} \mathrm{O}_{2} . \mathrm{MSN}-\mathrm{NA}\left(1 \mathrm{mg} \mathrm{mL}^{-1}\right.$ ) was photoirradiated at $665 \mathrm{~nm}$ for 0 (solid line) or $30 \mathrm{~min}$ (dotted line) in the presence of methylene blue $(100 \mu \mathrm{M})$. (B) Time dependence of phosphorescence emission $(1270 \mathrm{~nm})$ of ${ }^{1} \mathrm{O}_{2}$ generated from MSN-NEP (black) or NEP (red). (C) Chemiluminescent spectra of VPA (1.5 mM) in the presence of MSN-NEP (1 mg mL ${ }^{-1}$ ). After the mixture of VPA and MSN-NEP was left to stand for 90 min, the spectra were recorded under basic conditions (pH 11.0).

compared the lifetime of ${ }^{1} \mathrm{O}_{2}$ and confirmed that ${ }^{1} \mathrm{O}_{2}$ generated from MSN-NEP showed longer lifetime $(9.1 \mu \mathrm{s})$ than that from NEP $(4.8 \mu \mathrm{s})$ (Fig. 3B). These results and the evidence that the lifetime of ${ }^{1} \mathrm{O}_{2}$ is affected by their surrounding environment such as solvents ${ }^{17}$ led the conclusion that MSN-NEP thermally generated ${ }^{1} \mathrm{O}_{2}$ in their pores. We also confirmed that heating of MSN-NEP at $40{ }^{\circ} \mathrm{C}$ for $5 \mathrm{~min}$ resulted in an increase in absorption at around $300 \mathrm{~nm}$ due to the release of ${ }^{1} \mathrm{O}_{2}$ (Fig. S2 $†$ ). The number of naphthalene units incorporated on the surface of MSN was estimated to be $136 \mathrm{nmol} \mathrm{mg}{ }^{-1}$, which was calculated from the absorption at $290 \mathrm{~nm}$.

We then evaluated the reaction of VPA in the presence of MSN-NEP under basic conditions and compared the reaction efficiency between MSN-NEP and NEP upon reaction with VPA. HPLC analysis of the reaction showed that consumption amount of VPA in the presence of MSN-NEP was about half of that in the presence of NEP (Fig. S3†). Subsequently, we monitored the emission of VPA after the reaction for $1.5 \mathrm{~h}$ in the presence of MSN-NEP. Although the emission intensity was relatively weak, we observed chemiluminescence at around $450 \mathrm{~nm}$, as shown in Fig. 3C. These results indicate that ${ }^{1} \mathrm{O}_{2}$ was generated within the pores of MSN and reacted with VPA to form a dioxetane derivative, which degraded with the concomitant emission.

In conclusion, we prepared a novel chemiluminescent system using a vinyl phenol derivative (VPA) and naphthalene endoperoxide (NEP). In this system, VPA and NEP act as an emission unit and thermal ${ }^{1} \mathrm{O}_{2}$ generator, respectively. The combination of VPA and NEP resulted in the formation of a dioxetane derivative via the [2+2] cycloaddition of VPA and ${ }^{1} \mathrm{O}_{2}$; subsequent decomposition of a dioxetane derivative led to bright chemiluminescence. In addition, we prepared mesoporous silica nanoparticles bearing NEP within the pores (MSN-NEP) and confirmed that selective oxidation of VPA occurred within the pores to give a chemiluminescence. Although both the efficiency of the reaction and the emission intensity need to be improved, this approach may lead to a chemiluminescent emission system for biological imaging that does not require complex techniques such as gene or cellular manipulation. Further biological experiments are in progress.

\section{Acknowledgements}

We sincerely thank Professor Tadashi Suzuki (Aoyama Gakuin University) for the measurement of emission and lifetime of singlet oxygen. This work is partly supported by the Innovative Techno-Hub for Integrated Medical Bio-imaging Project of the Special Coordination Funds for Promoting Science and Technology, and by Grant-in-Aid for Scientific Research (for K. T. Grant number 23113508, for A. S. Grant number 16K12876), from the Ministry of Education, Culture, Sports, Science and Technology (MEXT), Japan.

\section{References}

1 A. Roda and M. Guardigli, Anal. Bioanal. Chem., 2012, 402, 69.

2 A. Roda, M. Guardigli, P. Pasini, M. Mirasoli, E. Michelini and M. Musiani, Anal. Chim. Acta, 2005, 541, 25.

3 R. Geiger, E. Shneider, K. Wallenfels and W. Miska, Biol. Chem. Hoppe-Seyler, 1992, 373, 1187.

4 W. Zhou, M. P. Valley, J. Shultz, E. M. Hawkins, L. Bernad, T. Good, D. Good, T. L. Riss, D. H. Klaubert and K. V. J. Wood, J. Am. Chem. Soc., 2006, 128, 3122.

5 M. P. Valley, W. Zhou, E. M. Hawkins, J. Shultz, J. J. Cali, T. Worzella, L. Bernad, T. Good, D. Good, T. L. Riss, D. H. Klaubert and K. V. Wood, Anal. Biochem., 2006, 359, 238.

6 H. Yao, M.-K. So and J. Rao, Angew. Chem., Int. Ed., 2007, 46, 7031.

7 W. Zhou, C. Andrews, J. Liu, J. W. Shultz, M. P. Valley, J. J. Cali, E. M. Hawkins, D. H. Klaubert, R. F. Bulleit and K. V. Wood, ChemBioChem, 2008, 9, 714.

8 A. Dragulescu-Andrasi, G. Liang and J. Rao, Bioconjugate Chem., 2009, 20, 1660.

9 M. Kindermann, H. Roschitzki-Voser, D. Caglic, U. Repnik, C. Miniejew, P. R. E. Mittl, G. Kosec, M. G. Grütter, B. Turk and K. U. Wendt, Chem. Biol., 2010, 17, 999.

10 G. C. Van de Bittner, E. A. Dubikovskaya, C. R. Bertozzi and C. J. Chang, Proc. Natl. Acad. Sci. U. S. A., 2010, 107, 21316.

11 A. S. Cohen, E. A. Dubikovskaya and C. R. Bertozzi, J. Am. Chem. Soc., 2015, 1966, 88. 
12 G. C. Van de Bittner, C. R. Bertozzi and C. J. Chang, J. Am. Chem. Soc., 2013, 135, 1783.

13 M. Matsumoto, J. Photochem. Photobiol., C, 2004, 5, 27.

14 A. P. Schaap, R. S. Handley and B. P. Giri, Tetrahedron Lett., 1987, 28, 935.

15 N. Hananya, A. E. Boock, C. R. Bauer, R. Satchi-Fainaro and D. Shabat, J. Am. Chem. Soc., 2016, 138, 13438.
16 T. Nakamura, A. Son, Y. Umehara, T. Ito, R. Kurihara, Y. Ikemura and K. Tanabe, Bioconjugate Chem., 2016, 27, 1058.

17 B. Rånby, J. F. Rabek, Singlet Oxygen: Reactions with organic compounds and polymers, John Wiley \& Sons, Chichester, 1978. 